\title{
Über die Wirkung verschiedener Pufferlösungen auf die Spirogyra-Zellen
}

Von

G. Yamaha und N. Suita

Aus dem Botanischen Institut der Tokyo-Universität für Literatur und Wissenschaft

(Mit 8 Abbildungen)

Eingegangen am 18 . Jalli 1939

Gelegentlich der Kultur- und Vitalfärbungsversuche an Spirogyra-Zellen fiel uns eine merkwürdige Tatsache auf, daß verschiedenartige Salze, wie sie auf gebräuchliche Nährflüssigkeiten sowie Pufferlösungen Anwendung finden, wenn jedes einzeln wirkt, auf die Spirogyra-Protoplasten nicht geringen schädigenden Einfluß ausüben. Es hat sich herausgestellt, daß diese Wirkung der Salze mit demselben Kation ( $\mathrm{K}$ ) bei demselben $\mathrm{pH}$ jenach der Anionenart in verschiedenen Maßen zur Entfaltung kommt. Es handelt sich also hierbei um Anionenwirkung. Im Gegensatz zur Kationenwirkung liegt uns bisher noch keine ausführliche diesbezügliche Angabe bei Spirogyra vor. Es scheint sich der Mühe zu lohnen, die Anionenwirkung bei diesem Material näher zu untersuchen.

\section{Methodisches}

Die zur Anwendung kommenden Spirogyren wurden im botanischen Garten unserer Universität gesammelt. Ihre spezifischen Namen waren unbestimmt. Sie gehören wenigstens zu 3 Arten von verschiedenen Zellgrößen. In den meisten Versuchen kamen nur die größtzelligen in Betracht, denen Gallertscheide fehlt (vgl. YaмAнA u. ARAKI 1939).

Geprïft wurden außer verschiedenen Sulfaten, folgende Arten der Pufferlösungen verschiedener Konzentration $(0,001,0,005,0,01$ und 0,05 GM) :

1. $\mathrm{KH}_{2} \mathrm{PO}_{4}+\mathrm{K}_{2} \mathrm{HPO}_{4}(\mathrm{pH} \quad 5,0 \quad 6,0 \quad 7,0 \quad 8,0, \quad 9,0)$.

2. Na-bzw. K-Azetat + Essigsäure ( $\mathrm{pH} 4,0 \quad 5,0,6,0, \quad 7,0)$.

3. Mono K-Citrat + $\mathrm{KOH} \mathrm{(} \mathrm{pH} 4,0,5,0,6,0)$.

4. Mono K-Phthalat $+\mathrm{KOH}(\mathrm{pH} 4,0,5,0,6,0)$.

Zur Herstellung der Lösungen wurde das aus Glas umdestillierte Wasser benutzt, in dem Spirogyren für gewöhnlich wenigstens 24 Stunden gesund leben. Aus dem Teichwasser wurden Spirogyra- 
Fïden 5-15 Minuten in das umdestillierten Wasser eingetaucht. Nach flüchtigem nochmaligen Spülen mit umdestilliertem Wasser ließen wir sie verschieden lang in Pufferlösungen verweilen. Mikroskopische Beobachtung erfolgte unter Anwendung von Kompensationsokular $20 \times$ oder $30 \times$, und apochr. Objektiv $20 \times, 40 \times$ oder achr. Objektiv $90 \times$ (ZEISS). Spirogyra-Fäden wurden immer mit einer zum Haken gebogenen Glasnadel aufgefangen.

\section{Verschiedene Arten der Strukturanomalien der Spirogyra-Protoplasten}

Wir möchten nun die Beschreibung verschiedener Strukturanomalien vorausschicken, wie sie Spirogyra-Zellen in verschiedenen Salzlösungen aufweisen.

1. Als das erste Stadium verschiedener Strukturanomalien der Spirog?ra-Zellen tritt häufig die Störung der spiraligen Anordnung der Chloroplasten auf (Abkürzung in der Tabelle-S).

2. Chloroplastentrennung (Abkürzung-T): Die Chloroplastenbänder lösen sich teilweise von dem Wandbelege des Zytoplasmas ab. Dabei wird das wandständige Zytoplasma zwischen dem Chloroplastenband und der Zellwand zu dünnen Strängen, breiten Brücken oder unregelmäßigen Schichten ausgezogen (vgl. SAKAMURA 1933, S. 302). Chloroplastentrennung tritt in der Regel als Vorläufer der Chloroplastenkontraktion in die Erscheinung.

3. Chloroplastenkontraktion (Abkürzung-K, vgl. GICKLHORN 1933) :

Chloroplastenbänder werden unter Beibehaltung spiraliger Anordnung ins Zellinnere oder an eine Seite der Zelle zusammengezogen. Dabei kommen im Allgemeinen Plasmafäden zum Vorschein, die sich zwischen dem Wandbelege und den Chloroplasten spannen (Abb. 2).

4. Chloroplastendisintegration (Abkürzung-D): Das erste Arzeichen für Chloroplastendisintegration besteht in der Oberflächenveränderung der Chloroplasten und zwar in der Regel in der Oberflächenverminderung (Abb. 3) und seltener Oberflächenvergrößerung. In normalem Zustand sind Chloroplastenränder gelappt bzw. gewellt und die Innenseite der Chloroplastenbänder ragt kielartig in den Zellsaftraum hinein (Abb. 1) (vgl. SAKAmura 1933, S. 291). Die Oberflächenverminderung der Chloroplasten kommt nun darin zum Ausdruck, daß die letzteren ganzrandig werden und die kielartigen Vorsprünge derselben verschwinden. Die Oberfläche der Chloroplasten kann dadurch vergrößert werden, daß ihre Ränder zackig werden und die Chloroplastenbänder seitlich miteinander anastomosieren, was bei nieder $€ \mathrm{~m} \mathrm{pH}$ der Lösungen häufig der Fall ist. 
Das nächstfolgende Stadium der Chloroplastendisintegration stellt entweder der tropfige Zerfall (Abb. 4) oder die Quellung (ohne Fragmentation) der Chloroplasten dar (vgl. ScHINDLER 1938, S. 192) (Abb. 5,6). Der tropfige Zerfall der Chloroplasten pflegt ohne Störung der spiraligen Anordnung derselben stattzufinden. Bei den alkalischen Lösungen wird die Quellung immer von der Vakuolisierung begleitet (Abb. 7, 8).

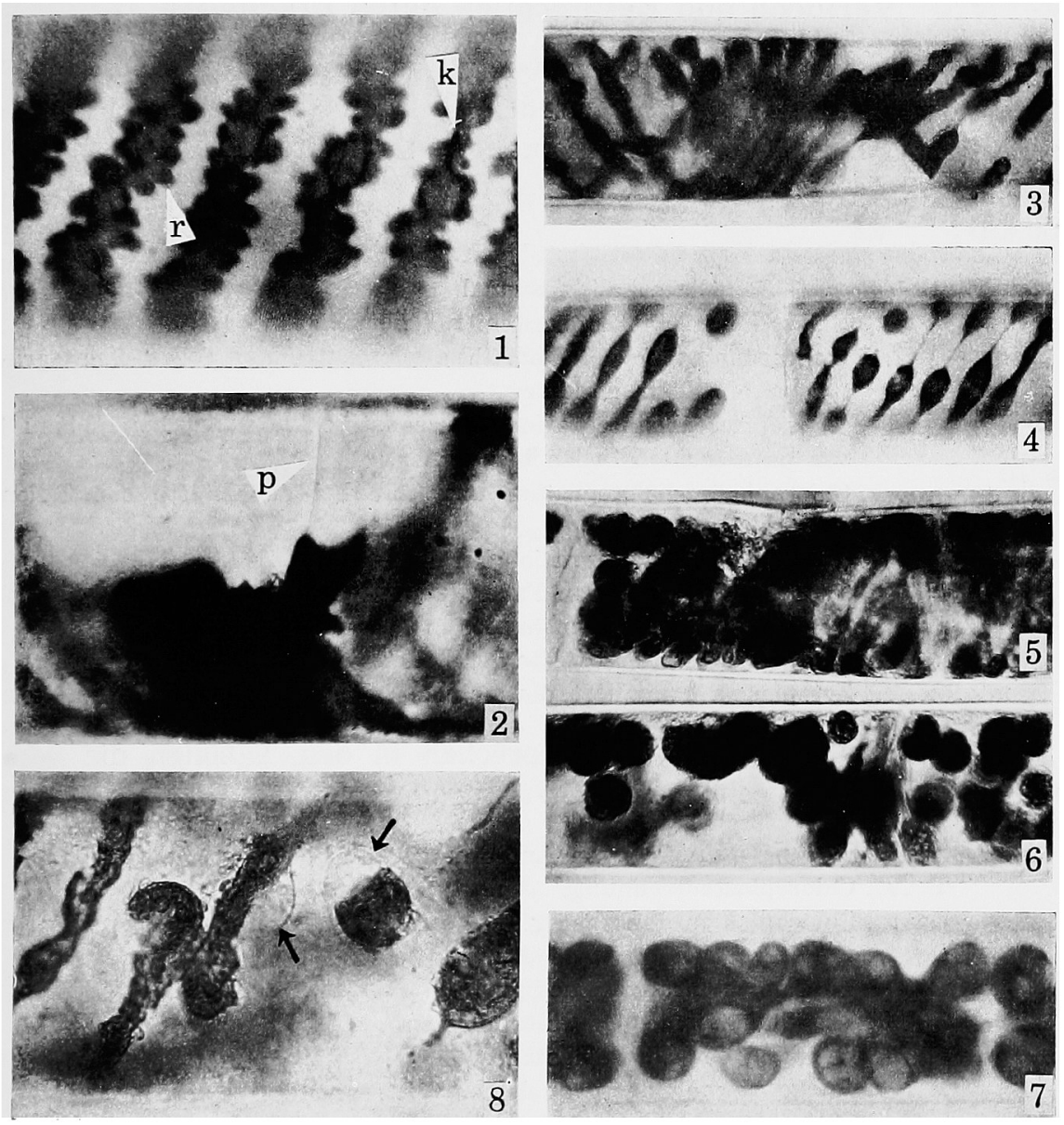

Abb. 1-8. Spirogyra sp. 1, INormale Chloroplastenbänder mit Randlappen (r) und kielartigen Vorsprüngen (k). 2, Chloroplastenkontraktion in 0.01 GM Phthalat-Puffer ( $\mathrm{pH} 8,8)$ (60 Minuten). p, Plasmafaden gespannt zwischen dem Chloroplastenband und der Zellwand. 3, Früheres Stadium der Chloroplastendisintegration in 0,02 GM Zitratpuffer ( $\mathrm{pH} 4,5$ ) (30 Minuten). 4, Tropfiger Zerfall der Chloroplasten in 0,02 GM Zitratpuffer ( $\mathrm{pH} 4,5)$ (60 Minuten). 5, Chloroplastendisintegration in 0,01 GM Phcsphatpuffer ( $\mathrm{pH} 8,0)$ (4 Stunden). 6, Tropfiger Zerfall der Chloroplasten unter Quellung in 0,01 GM Phosphatpuffer ( $\mathrm{pH} 8,0$ ) (4 Stunden). 7, Vakuolisierung gequollene $\mathbf{r}$ Chloroplasten in 0,01 GM Phosphatpuffer ( $\mathrm{pH} \mathrm{7,8)} \mathrm{(60} \mathrm{Minuten).} \mathrm{8,} \mathrm{Blasenförmige}$ Vakuolisierung der Chloroplasten in 0,02 GM Zitratpuffer ( $\mathrm{pH} 4,6$ ) (60 Minuten)• Vergrößerung: 1. $540 \times, 2,8.450 \times, 3-7.225 \times$. 
Das normale Zytoplasma zeigt Mikrosomen, die Brownscher Bewegung oder lebhafter Strömung unterworfen sind. Der lebende Zellkern besitzt eine längliche Gestalt. Sein verschwommener Umriß erscheint in geschädigtem Zustand deutlich. Die Form des gestorbenen Kerns ist meistens rundlich.

\section{Versuche und Besprechung deren Ergebnisse}

Die Versuchsergebnisse sollen unten übersichtlichkeitshalber tabellarisch zusammengestellt werden. In den Tabellen werden verschiedene Grade der Strukturanomalien mit dem Zeichen + bemerkt. Größore Zahl von + zeigt erhöhte Wirkung betreffender Lösung. So z.B :

- bedeutet.... Sämtliche Zellen normal.

$\pm \quad \ldots$... Einige Zellen mit ungeordneten Chloroplasten.

$+\quad \ldots$ Ungeordnete Chloroplasten, Chloroplastentrennung oder -disintegration vereinzelt bemerkbar.

$++\ldots$ Chloroplastendisintegration oder sonstige Anomalien bei $1 / 5$ bis $1 / 3$ von untersuchten Zellen bemerkbar.

$+++\ldots$ Chloroplastendisintegration ungefähr bei der Hälfte der Zellen bemerkbar.

$++++\ldots$ Chloroplastendisintegration bei den meisten Zellen.

$x \quad$.... Zytoplasma bei einigen Fäden koaguliert $u$. fixiert.

$\times \times \quad \ldots$. Zytoplasma bei noch mehr Zellen koaguliert $u$. fixiert.

$x \times x \quad \ldots$ Zytoplasma bei der Hälfte der Zellen koaguliert $u$. fixiert.

$x \times x \times \ldots$ Zytoplasma koaguliert, die meisten Zellen im fixierten Zustand tot.

Folgenden Abkürzungen entsprechen in den Tabellen die verschiedenen Arten beobachteter Strukturanomalien:

S .... Störung der spiraligen Anordnung der Chloroplasten.

T .... Chloroplastentrennung.

Dg.... Cbloroplasten ganzrandig.

Dq.... wie oben, aber mit Andeutung an die Quellung

$\mathrm{Dz}$.... Chloroplastenränder zackig.

Z .... Tropfiger Zerfall der Chloroplasten.

Q .... Quellung der Chloroplasten.

V .... Vakuolisierung der gequollenen Chloroplasten.

Dk.... Chloroplastendisintegration unter Kontraktion.

K .... Chloroplastenkontraktion.

Tabelle 1. K-Phosphat $(0,005 \mathrm{GM})$ :

\begin{tabular}{|c|c|c|c|c|c|c|c|}
\hline Min. & $\mathrm{pH}$ & 5,0 & 6,0 & 7,0 & 7,4 & 8,0 & 9,0 \\
\hline 30 & & - & - & - & + & + & $+t+t$ \\
\hline 60 & & $\stackrel{ \pm}{\mathrm{T}}$ & $\stackrel{ \pm}{T}$ & $\mathrm{~T}, \stackrel{+}{\mathrm{Dg}}, \mathrm{Dq}$ & $+t$ & $\mathrm{Dq}^{++}, \mathrm{Q}, \mathrm{V}$ & $\begin{array}{l}++++ \\
\mathrm{Dq}, \mathrm{Q}, \mathrm{V}\end{array}$ \\
\hline
\end{tabular}


Tabelle 2. K-Phophat $(0,01 \mathrm{GM})$ :

\begin{tabular}{|c|c|c|c|c|c|c|}
\hline Min. & $\mathrm{pH}$ & 5,0 & 6,0 & 7,0 & 8,0 & 9,0 \\
\hline 20 & & - & + & $\mathrm{T}, \stackrel{+}{+}, \mathrm{Dq}$ & $\mathrm{T}, \mathrm{Dg}, \stackrel{+}{\mathrm{Dq}}, \mathrm{Q}$ & $\mathrm{T}, \stackrel{++}{\mathrm{Dg},}, \stackrel{+}{\mathrm{D} q}, \mathrm{Q}$ \\
\hline 30 & & + & + & ++ & & \\
\hline 60 & & + & ++ & $\mathrm{T}, \stackrel{++++}{\mathrm{Dq}}, \mathrm{Q}, \mathrm{V}$ & $\underset{\mathrm{Q}, \mathrm{V}}{++}$ & $\underset{\mathrm{Q}, \mathrm{V}}{++}$ \\
\hline 180 & & $\mathrm{Dg}, \mathrm{Dq}$ & $\stackrel{++}{\mathrm{Dg}, \stackrel{+}{\mathrm{Dq}}}$ & $\begin{array}{l}++++ \\
\mathrm{Dq}, \mathrm{Q}, \mathrm{V}\end{array}$ & & \\
\hline
\end{tabular}

Tabelle 3. K-Phosphat $(0,05 \mathrm{GM})$ :

\begin{tabular}{|c|c|c|c|c|c|c|c|c|}
\hline Min. & $\mathrm{pH} \quad 4,7$ & 5,0 & 6,0 & 7,0 & 7,4 & 8,0 & 8,6 & 9,0 \\
\hline 10 & + & $\stackrel{+}{D_{q}}$ & ++ & $\mathrm{s}, \stackrel{ \pm}{\mathrm{T}}$ & & $\mathrm{K}, \stackrel{+}{\mathrm{D} k}$ & \pm & \\
\hline 20 & ++ & ++ & +++ & ++ & & & & \\
\hline 30 & $++t$ & $\stackrel{+}{++}+\stackrel{+}{K}, \mathrm{Dk}$ & ++++ & ++ & & + & + & \\
\hline 60 & & $\begin{array}{l}++++ \\
\mathrm{Dg}, \stackrel{+}{\mathrm{Dq}}\end{array}$ & $\begin{array}{l}++++ \\
\mathrm{Dg}, \mathrm{Dq}\end{array}$ & ++ & $\mathrm{Dg}^{+} \mathrm{Dz}$ & $\mathrm{Dg}, \stackrel{+}{\mathrm{D}} \mathrm{z}, \mathrm{Z}$ & & $\mathrm{Dg}, \stackrel{+}{\mathrm{D}} z, \mathrm{Z}$ \\
\hline 120 & & ++++ & ++++ & $\stackrel{++}{\mathrm{Dg}, \stackrel{+}{\mathrm{D} q}}$ & & ${ }_{\mathrm{Dq}}^{+} \mathrm{Q}$ & $\mathrm{T}, \stackrel{+}{\mathrm{Dg}}, \mathrm{Dq}$ & \\
\hline 200 & & ++++ & ++++ & ++++ & $\stackrel{++++}{++}$ & $\underset{\mathrm{Dq}, \mathrm{Q}}{++++}$ & $\underset{\mathrm{Dq}, \mathrm{Q}}{+++}$ & $\underset{\mathrm{Dq}, \mathrm{Q}}{+++}$ \\
\hline
\end{tabular}

Tabelle 4. Na-Azetat (0,001 GM) :

\begin{tabular}{c|ccccc}
\hline Min. & $\mathrm{pH}$ & 4,0 & 5,0 & 6,0 & 7,0 \\
\hline 10 & & + & + & \pm & - \\
30 & & $++\mathrm{Dq}, \mathrm{Dk}$ & $+\mathrm{T}, \mathrm{K}$ & \pm & - \\
60 & & ++++ & ++ & \pm & - \\
\hline
\end{tabular}

Na-Azetat $\quad(0,005$ GM) :

\begin{tabular}{c|cccc}
\hline Min. & $\mathrm{pH}$ & 4,0 & 5,0 & 6,0 \\
\hline 30 & & $++++\mathrm{Dq}$ & $++++\mathrm{K}, \mathrm{Dq}$ & +
\end{tabular}

Tabelle 5. Na-Azetat $(0,01 \mathrm{GM})$ :

\begin{tabular}{c|ccccc}
\hline Min. & $\mathrm{pH}$ & 4,0 & 5,0 & 6,0 & 7,0 \\
\hline 10 & $++\mathrm{T}, \mathrm{K}, \mathrm{Dq}$ & $++\mathrm{Dk}$ & $\pm \mathrm{S}$ & \\
20 & & $+++\mathrm{T}, \mathrm{K}, \mathrm{Z}, \mathrm{Dq}$ & $+++\mathrm{Z}, \mathrm{Dq}, \mathrm{Dk}$ & + & \\
30 & & $\times \times \times$ & $\times \times \times \times$ & $+\mathrm{T}$ & \\
60 & $\times \times \times \times$ & $\times \times \times \times$ & $+\mathrm{T}$ & $+\mathrm{S}, \mathrm{T}$ \\
90 & $\times \times \times \times$ & $\times \times \times \times$ & &
\end{tabular}


Tabelle 6. K-Zitrat $(0,001 \mathrm{GM})$ :

\begin{tabular}{c|cccc}
\hline Min. & pH & 4,0 & 5,0 & 6,0 \\
\hline 10 & & $\pm \mathrm{S}, \mathrm{T}$ & $+\mathrm{T}, \mathrm{Dg}$ & - \\
30 & & $++\mathrm{T}, \mathrm{Dk}$ & $++\mathrm{T}, \mathrm{Dg}$ & - \\
60 & & $+++\mathrm{T}, \mathrm{K}, \mathrm{DK}$ & $++\mathrm{T}, \mathrm{Dk}, \mathrm{Dq}$ & -
\end{tabular}

Tabelle 7. K-Zitrat $(0,01 \mathrm{GM})$ :

\begin{tabular}{|c|c|c|c|c|c|}
\hline Min. & $\mathrm{pH}$ & 4,0 & 5,0 & 6,0 & \\
\hline 10 & & $\times \times \times \times \mathrm{T}, \mathrm{Dg}$ & $++\quad \mathrm{S}, \mathrm{K}, \mathrm{Z}, \mathrm{Dq}, \mathrm{Q}$ & + & $\mathrm{K}, \mathrm{Z}, \mathrm{Dq}, \mathrm{Q}$ \\
\hline 30 & & $\times \times \times \times \mathbf{T}, \mathrm{Dg}, \mathrm{Dq}, \mathrm{Dk}$ & $++++\mathrm{Z}, \mathrm{Dq}, \mathrm{Q}$ & + & $\mathrm{S}, \mathrm{Dq}, \mathrm{Q}$ \\
\hline 60 & & $\times \times \times \times \mathrm{T}, \mathrm{Dg}, \mathrm{Dk}$ & $++++\mathrm{Z}, \mathrm{Dq}, \mathrm{Q}$ & $++t$ & - Dq, Q \\
\hline
\end{tabular}

Tabelle 8. K-Zitrat $(0,05 \mathrm{GM})$ :

\begin{tabular}{c|cccc}
\hline Min. & pH & 4,0 & 5,0 & 6,0 \\
\hline 10 & & $+\mathrm{T}$ & - & - \\
30 & & $\times \times \times \times$ & & $++++\mathrm{Dq}, \mathrm{Dk}$ \\
93 & & $\times \times \times \times$ & $++++\mathrm{S}, \mathrm{Z}, \mathrm{Q}$ & +
\end{tabular}

Tabelle 9. K-Zitrat $(0,1 \mathrm{GM})$ :

\begin{tabular}{c|cccl}
\hline Min. & $\mathrm{pH}$ & 4,0 & 5,0 & 6,0 \\
\hline 30 & & $\times \times \times \times$ & $\mathrm{Dz}$ & $\mathrm{Dz}$
\end{tabular}

Tabelle 10. K-Phthalat (0,001 GM):

\begin{tabular}{|c|c|c|c|c|}
\hline Min. & $\mathrm{pH}$ & 4,0 & 5,0 & 6,0 \\
\hline 10 & & $++\mathrm{T}, \mathrm{K}$ & $\pm \mathrm{T}$ & - \\
\hline 30 & & $+++\mathrm{T}, \mathrm{Dq}, \mathrm{Dk}$ & $+\mathrm{T}$ & $\pm \mathrm{T}$ \\
\hline 60 & & $++++\mathrm{T}, \mathrm{K}, \mathrm{Dq}$ & $++\mathrm{S}, \mathrm{T}, \mathrm{K}$ & $\pm \mathrm{S}$ \\
\hline 120 & & $++++\mathrm{T}, \mathrm{K}, \mathrm{Dq}$ & $++\mathrm{T}, \mathrm{K}$ & $\pm \mathrm{S}$ \\
\hline
\end{tabular}

Tabelle 11. K-Phthalat $(0,01 \mathrm{GM})$ :

\begin{tabular}{|c|c|c|c|}
\hline Min. & $\mathrm{pH}$ & 5,0 & 6,0 \\
\hline 10 & $+++\mathrm{T}, \mathrm{K}, \mathrm{Dk}, \mathrm{Dg}, \mathrm{Dq}$ & $++\mathrm{T}, \mathrm{Dq}$ & $\pm \mathrm{T}$ \\
\hline 30 & $\times \times \times \times++++\mathrm{T}, \mathrm{Dq}, \mathrm{Dk}$ & $++\mathrm{T}, \mathrm{Dq},(\mathrm{Q})$ & $++\mathrm{T}, \mathrm{K}, \mathrm{Dq}$ \\
\hline 60 & $\times \times \times \times$ & $++\mathrm{T}$ & $++\mathrm{S}, \mathrm{K}$ \\
\hline 120 & & $++\mathrm{T}, \mathrm{S}$ & \\
\hline 200 & & $++\mathrm{T}, \mathrm{S}$ & $++\mathrm{T}, \mathrm{Dq}$ \\
\hline
\end{tabular}


Tabelle 12. K-Phthalat $(0,05 \mathrm{GM})$ :

\begin{tabular}{r|cccc}
\cline { 2 - 5 } Min. & $\mathrm{pH}$ & 4,0 & 5,0 & 6,0 \\
\hline $\mathbf{5}$ & & $\times \times \times \times \mathrm{Dz}$ & $+\mathrm{T}$ & $+++\mathrm{T}, \mathrm{Dq}$ \\
15 & & $\times \times \times \times \mathrm{Dz}, \mathrm{S}$ & $++\mathrm{Dk}, \mathrm{Dq}$ & $++++\mathrm{T}, \mathrm{Dq}, \mathrm{Q}$ \\
30 & & & $++++\mathrm{Dk}, \mathrm{Dq}$ &
\end{tabular}

Bei der Durchsicht der obenstehenden Tabellen ist vor allem zu ersehen, daß die schädigende Wirkung der Pufferlösungen nicht weniger auf ihrem pH-Wert als auf der Art und der GM-Konzentration derselben abhängig ist. Wie man theoretisch leicht erwarten kann, so tritt die Anionenwirkung jeder Pufferlösung im. Vergleich zur H-Ionenwirkung um so deutlicher in den Vordergrund, je höher die GM-Konzentration derselben ist. Demgemäß wird der Wirksamkeitsunterschied einer und derselben Art der Pufferlösungen von verschiedenen $\mathrm{pH}$ voneinander bei höheren Konzentrationen minimal (vgl. Tabelle 8, 9, 11 usw.). Besonders auffallend fällt diese Anicnenwirkung in die Augen bei Phosphatpufferlösungen, die im weiteren $\mathrm{pH}$ - Umfange ( $\mathrm{pH} 5,0-9,0)$ zur Anwendung kommen können. Phosphationen wirken eigentlich auf Protoplasten anscheinend entquellend.") Dementsprechend wirken sie den quellend wirkenden $\mathrm{OH}-$ Ionen gegenüber antagonistisch. Je höher das $\mathrm{pH}$ der Pufferlösung wird, umso ausgesprochener tritt die Quellungsanomalien der Chloroplasten ein, die aber bei der höheren Konzentration der Pufferlösung von demselben $\mathrm{pH}$ immer seltener zum Vorschein kommt (vgl. Tabelle 1-3). Diese entquellende Wirkung der Anionen kommt auch bei Zitratpufferlösung in einem geringeren Maßen zur Geltung (vgl. Tabelle 6-8). Die Azetatpufferlösung zeichnet sich dadurch aus, $\mathrm{da}$ sie bei $\mathrm{pH}$ 4,0 und 5,0 auf Protoplasma stark koagulierend wirkt, während bei $\mathrm{pH}$ 6,0 die schädigende Wirkung bedeutend geschwächt wird. Diese starke koagulierende Wirkung der Azetatpufferlösung auf Protoplasma erfahren wir auch bei den Pollenmutterzellen (YAMAHA 1938). Es handelt sich hierbei ohne Zweifel eigentlich um H-Ionenwirkung. Azetationen selbst scheint nicht so große Wirksamkeit zuzukommen, wie es gleich unten gezeigt wird (vgl. weiter SAKAMURA 1934). Nach SaKamura (a.a.0.) wirken Na-Azetat und K-Azetat quantitativ voneinander etwas verschieden. Es scheint also wünschenswert zu sein, bei unserem Material die Wirkung beider Azetate miteinander zu vergeichen. Aus den Versuchsergebnissen geht aber hervor, daß K-Azetat im Gegensatz zur Angabe SAKA-

1) Nach PaULI gilt bei der Koagulation des natürlichen Ovalbumins folgende Anionenreihe :

$\mathrm{SCN}<\mathrm{J}<\mathrm{Br}<\mathrm{NO}_{3}<\mathrm{Cl}<$ Azetat $<$ Tartrat $<$ Zitrat $<\mathrm{PO}_{4}<\mathrm{SO}_{4}<\mathrm{F}$ 
MURAs, etwas wirksamer ist als $\mathrm{Na}$-Azetat, wie die untenstehende Tabelle veranschaulicht:

Tabelle 13. K-Azetat $(0,01 \mathrm{GM})$ :

\begin{tabular}{|c|c|c|c|c|}
\hline Min. & $\mathrm{pH}$ & $3,95-3,90$ & 4,95 & 5,9 \\
\hline 10 & & $\times \times \times \times \mathrm{Dz}$ & & \\
\hline 30 & & wie oben & $x \times++\mathrm{Dg}, \mathrm{Dz}$ & $++\mathrm{S}, \mathrm{T}, \mathrm{K}, \mathrm{Dq}$ \\
\hline 60 & & wie oben & $x \times++\mathrm{Dg}, \mathrm{Dz}, \mathrm{Dq}$ & $+++\mathrm{S}, \mathrm{T}, \mathrm{K}, \mathrm{Dq}$ \\
\hline \multicolumn{5}{|c|}{ Na-Azetat $\quad(0,01 \mathrm{GM})$ : } \\
\hline Min. & $\mathrm{pH}$ & $3,8-3,9$ & 4,95 & 6,0 \\
\hline 10 & & $\times \times \times \times \mathrm{Dz}$ & & \\
\hline 30 & & wie oben & $x+++\mathrm{K}, \mathrm{Dk}, \mathrm{Dq}$ & $++\mathrm{S}, \mathrm{T}, \mathrm{K}, \mathrm{Dq}$ \\
\hline 60 & & wie oben & $x+++D q$ & $++\mathrm{S}, \mathrm{T}, \mathrm{K},(\mathrm{Dq})$ \\
\hline
\end{tabular}

Um weiterhin über die relative Wirksamkeit verschiedener Anionen klar zu werden, wurden eine besondere Reihe von Versuchen angestellt und zwar mit 0,01 und 0,001 GM Pufferlösungen von $\mathrm{pH}$ 4,0, 5,0 und 6,0. Die Versuchsresultate werden in Tabellen 14 und 15 zusammengestellt. Daraus ergibt sich ohne weiteres folgende Wirksamkeitsreihe der Anionen:

$$
\text { Zitrat }>\text { Phosphat }>\text { Azetat }>\text { Phthalat }
$$

Tabelle 14. Konzentration $0,001 \mathrm{GM}$, Temperatur $27,5^{\circ} \mathrm{C}$

\begin{tabular}{l|ccccc}
\hline & pH & 4,0 & 5,0 & 6,0 \\
& Min. & 60 & 60 & 60 und 120 Stunden \\
\hline K-Azetat & & $++\mathrm{T}, \mathrm{Dk}, \mathrm{Dg}$ & $+\mathrm{T}, \mathrm{K}$ & - & $\pm \mathrm{S}$ \\
K-Phthalat & & $++++\mathrm{K}, \mathrm{Dk}, \mathrm{Dg}$ & $+\mathrm{S}, \mathrm{T}, \mathrm{K}$ & - & $\pm \mathrm{S}$ \\
K-Phosphat & & $+++\mathrm{T}, \mathrm{K}, \mathrm{Dk}, \mathrm{Dq}$ & $+\mathrm{T}, \mathrm{K}$ & - & $\pm \mathrm{S}$ \\
K-Zitrat & & $++++\mathrm{K}, \mathrm{Dk}$ & $++\mathrm{T}, \mathrm{K}$ & $+\mathrm{T}$ & $++\mathrm{T}, \mathrm{K}, \mathrm{Dq}$
\end{tabular}

Tabelle 15. Konzentration $0,01 \mathrm{GM}$, Temperatur $26^{\circ} \mathrm{C}$, 30 Minuten

\begin{tabular}{|c|c|c|c|}
\hline & $\mathrm{pH} \quad 4,0$ & 5,0 & 6,0 \\
\hline K-Azetat & $\times \times \times \times \mathrm{Dg}, \mathrm{Dz}$ & 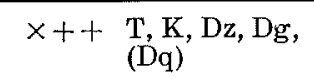 & $+\underset{(\mathrm{Dg})}{\mathrm{S}, \mathrm{T}, \mathrm{K}, \mathrm{Dk}}$ \\
\hline K-Phthalat & $x+++\mathrm{Dg}, \mathrm{Dk}$ & $++++\mathrm{T}, \mathrm{Dk}, \mathrm{Dq}$ & $+\mathrm{S}, \mathrm{T}, \mathrm{K}$ \\
\hline K-Phosphat & $x+++\mathrm{Dk}, \mathrm{Dq}$ & $++++\mathrm{K}, \mathrm{Dq}, \mathrm{Q}$ & $++\mathrm{T}, \mathrm{Dq}, \mathrm{Q}$ \\
\hline K-Zitrat & $\times x++\mathrm{Dk}, \mathrm{Dg}, \mathrm{Dz}$ & $x+++D q,(Q)$ & $++++\mathrm{Dq}, \mathrm{Q}$ \\
\hline
\end{tabular}

Tabelle 15 macht es höchst wahrscheinlich, daß die obenstehende Ionenreihe auch für die quellende Wirkung der Pufferlösungen auf 
Chloroplasten zur Geltung kommt. $\operatorname{Im}$ zu sehen, welchen Einfluß verschiedene Pufferlösungen auf die Quellung der Gelatinegallerte ausüben, wurden eine Reihe von Modellversuchen angestellt. Die Streifen aus Gelatinegallert $(4 \times 0,5 \times 0,2 \mathrm{~cm})$ wurden in $0,1 \mathrm{GM}$ Pufferlösungen von $\mathrm{pH} 6,0$ bei $22-23^{\circ} \mathrm{C} 16$ Stunden eingetaucht. Verlängerung der Gallertstreifen durch Quellung betrug wie folgt:

$\begin{array}{lc}\text { Pufferlösung } & \text { Verlängerung der Gelatinestreifen in } \% \\ \text { K-Azetat } & 59,4 \\ \text { K-Phthalat } & 79,4 \\ \text { K-Phosphat } & 68,7 \\ \text { K-Zitrat } & 75,0\end{array}$

Dabei gilt also als Quellungsreihe:

$$
\text { Phthalat }>\text { Zitrat }>\text { Phosphat }>\text { Azetat }
$$

Mit Ausnahme von Phthalat gehen somit die Quellung der Gelatinegallerte und Chloroplastendisintegration durch verschiedene Pufferlösungen (wenigstens bei $\mathrm{pH}$ 6,0) miteinander genau in Parallele.

Nach BenEcke (1907), SAKamura (1922, 1934) und Eiselsberg (1937) entfalten Ca-Ionen bei verschiedenartigen schädigenden Lösungen (angesäuerten Lösungen, NaCl-, $\mathrm{KCl}$-, LiCl- und $\mathrm{Na}$ Azetat-Lösung usw.) auf Spirogyra-Zellen entgiftende Wirkung. Untenstehende Tabelle zeigt einwandfrei, wie auch bei unseren Pufferlösungen Ca-Ionen schädliche Wirkung vermindern können.

Tabelle 16. 0,01 GM Pufferlösungen, $\mathrm{CaCl}_{\mathfrak{q}}$-Zusatz von $0,001 \mathrm{n}$

\begin{tabular}{|c|c|c|}
\hline & 1 Std. & 24 Std. \\
\hline 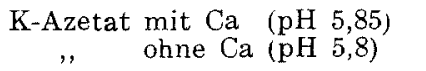 & $\bar{\dagger} \mathrm{S}, \mathrm{T}, \mathrm{K}$ & $\overline{+}+\mathrm{Z}, \mathrm{Dq}, \mathrm{Q}$ \\
\hline 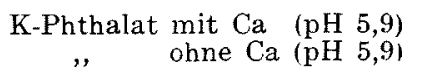 & $\begin{array}{l}\mathrm{S} ? \\
\mathrm{~S}, \mathrm{~T}\end{array}$ & $\begin{array}{ll} \pm & S \\
+t & \mathrm{Dq}\end{array}$ \\
\hline $\begin{array}{cll}\text { K-Phosphat mit } & \mathrm{Ca}(\mathrm{pH} 5,8) \\
,, & \text { ohne } \mathrm{Ca}(\mathrm{pH} 5,8)\end{array}$ & $+\overline{+}+\mathrm{Dk}, \mathrm{Dq}, \mathrm{Z}, \mathrm{Q}$ & $\overline{+}++\mathrm{Dq}, \mathrm{Z}, \mathrm{Q}$ \\
\hline 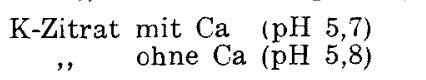 & $\begin{array}{l}++\mathrm{S}, \mathrm{T}, \mathrm{Dq},(\mathrm{Q}) \\
+++\mathrm{Dq}, \mathrm{Q}\end{array}$ & $\begin{array}{l}++++D k, D q \\
++++D q, Z, Q\end{array}$ \\
\hline
\end{tabular}

In Tabelle 17 können wir die relative Wirksamkeit von $\mathrm{KCl}, \mathrm{CaCl}_{2}$ und $\mathrm{MgCl}_{z,}$, wie sie als Zusatz in den Pufferlösungen vorhanden sind, übersehen. Wir bemerken, daß $\mathrm{KCl}$ bei Zusatz von $0,00 \mathrm{ln}$ die schädliche Wirkung von 0,01GM Phosphat-Pufferlösung von $\mathrm{pH} 5,7$ noch merklich verstärkt, während sie durch $\mathrm{MgCl}_{\text {.2 }}$ ziemlich und durch $\mathrm{CaCl}_{2}$ bedeutend vermindert wird. 
Tabelle 17. Phosphat-Puffer $0,01 \mathrm{GM}, \mathrm{pH} 5,6-5,7$, Temperatur $29^{\circ} \mathrm{C}$

\begin{tabular}{l|c}
\hline K-Phosphat ohne Zusatz: & $+++\mathrm{T}, \mathrm{Dk}, \mathrm{Dq}, \mathrm{Z}, \mathrm{Q}$ \\
K-Phosphat mit $\mathrm{KCl}(0,001 \mathrm{n})$ & $+++\mathrm{K}, \mathrm{Dq}, \mathrm{Z}, \mathrm{Q})^{1)}$ \\
K-Phosphat mit $\mathrm{CaCl}_{2}(0,002 \mathrm{n})$ & $-\mathrm{S} ?$ \\
K-Phosphat mit $\mathrm{MgCl}_{2}(0,002 \mathrm{n})$ & $++\mathrm{Dg}, \mathrm{Gq}, \mathrm{Z}, \mathrm{Q}$ \\
\hline
\end{tabular}

Anhangsweise möchten wir hier auch die zufällig beobachtete eigentümliche Wirkung von $\mathrm{SO}_{4}$-Ionen auf Spirogyra-Protoplasma zusammenfassend berichten. Merkwürdig sei nämlich, daß Spirogyra-Zellen zwar mit 0,2 $\mathrm{GM} \mathrm{MgCl}_{2}$ und $0,6 \mathrm{GM}$ Rohrzucker deutlich sich plasmolysieren lassen, aber mit $0,2 \mathrm{GMI}_{\mathrm{Na}} \mathrm{SO}_{4}$ und $0,3 \mathrm{GM}$ $\mathrm{MgSO}_{4}$ nicht plasmolysierbar sind. Die sämtlichen geprüften Sulfate $\left(\mathrm{Na}_{2} \mathrm{SO}_{4}, \mathrm{MgSO}_{4}\right.$ und $\left.\mathrm{CaSO}_{4}\right)$ sind darin einig, daß sie auch in verdünnter Konzentration (hypotonisch) immer deutliche Chloroplastenkontraktion verursachen können. Es hat den Anschein, als ob beide Erscheinungen irgendwie kausal verknüpft sind. Ähnlichen Eindruck haben wir auch bei der Saponinwirkung auf Spirogyra-Zellen gemacht (vgl. YAMAHA u. ARAKI 1939). Wenn man die 15-20 Minuten mit 0,3 GM $\mathrm{MgSO}_{4}$ behandelten Spirogyra-Zellen in 0,6 GM Rohrzucker einlegt, so bemerkt man die schwache eckige Plasmolyse, was auf die veränderte Beschaffenheit des Protoplasmas hindeutet.

\section{Zusammenfassung}

1. Verschiedenartige Pufferlösungen von $\mathrm{pH} 4,0-9,0$ bei der Konzentration von 0,001-0,05 GM bedingen verschiedene Strukturanomalien von Chloroplasten und Zytoplasma.

2. Diese schädliche Wirkung der Salzlösungen, an der neben $\mathrm{H}$ - und $\mathrm{OH}-$ Ionen auch Anionen wesentlich teilnehmen, läßt sich durch $\mathrm{Ca}$ - und Mg-Ionen in verschiedenen Maßen vermindern (Entgiftung).

3. Relative Wirksamkeit der verschiedenen Pufferlösungen von demselben $\mathrm{pH}$ geht mit der quellenden Wirkung auf Gelatinegallerte in Parallele. Dabei macht Phthalat-Puffer eine Ausnahme.

4. Es hat sich wenigstens bei Phosphat- sowie Zitrat-Pufferlösungen gezeigt, daß die Anionen in den Pufferlösungen der quellenden Wirkung von OH-Ionen anscheinend entgegenwirken, so daß die höhere Konzentration der Pufferlösung gleichsam wie die Erniedri gung des pH derselben wirkt (vgl. YamaHA u. IsHII 1932).

5. Sulfat-Ionen machen Spirogyra-Zellen schwer plasmolysierbar und bedingen Chloroplastenkontraktion. 
1940 U̇ber die Wirkung verschiedener Pufferlösungen auf die Spirogyra-Zellen 381

Zum Schluß möchten wir der Japanischen Gesellschaft zur Förderung der Zytologie für ihre finanzielle Unterstützung an dieser Untersuchungen unseren aufrichtigsten Dank aussprechen.

\section{Schriftenverzeichnis}

Benecke, W. (1907) Ber. d. d. bot. Ges. 25 : 322.

Eiselsberg, C. v. (1937) Biologia Generalis $13: 529$.

Gicklhorn, J. (1933) Protoplasma 17: 571.

Sakamura, T. (1922) Bot. Mag. Tokyo 36: 133.

- (1933) J. Fac. Sci. Hokkaido Imp. Univ. V. 2 : 287.

- (1934) Ebenda 3: 103.

Schindler, H. (1938) Protop'asma 30: 186.

Yamaha, G. (1938) Sci. Rep. Tokyo Bunrika Daigaku B 3 : 279.

- u. Araki, Z. (1989) Ebenda 4: 129. 